\title{
Labyrinth Absorber based on Metageometries Metasurface for Fungi Detection
}

\author{
Irati Jáuregui-López ${ }^{1,2}$, Pablo Rodriguez-Ulibarri ${ }^{1}$, Sergei A. Kuznetsov ${ }^{3,4}$, Miguel Beruete ${ }^{1,2}$ \\ ${ }^{1}$ Antennas Group-TERALAB: Electric and Electronic Engineering Department, Universidad Pública de Navarra, \\ Pamplona, Spain, miguel.beruete@unavarra.es * \\ ${ }^{2}$ Multispectral Biosensing Group, Navarrabiomed, Complejo Hospitalario de Navarra (CHN), Universidad Pública de Navarra \\ (UPNA), IdiSNA. Irunlarrea 3, 31008 Pamplona, Navarra, Spain \\ ${ }^{3}$ Rzhanov Institute of Semiconductor Physics SB RAS, Novosibirsk Branch "TDIAM", Lavrentiev Ave. 2/1, 630090 Novosibirsk, \\ Russia \\ ${ }^{4}$ Novosibirsk State University, Pirogova St. 2, 630090 Novosibirsk, Russia
}

\begin{abstract}
In this paper a labyrinth metasurface based in the new paradigm of metageometries is designed to operate in the Terahertz (THz) band as a biosensor. First, a numerical study is carried out to study the performance of the metasurface as a refractometer when working in two different configurations: transmission and reflection. Then, its performance as a fungi detector is evaluated and a comparison with other devices is performed, showing that the sensitivity and Figure of Merit (FOM) can be enhanced by the use of these kind of devices, in comparison with the classical approach of metaatoms. Particularly, the designed structure is able to detect 5 fungi elements arbitrarily distributed on the unit cell, which is equivalent to a concentration of $0.004 / \mu \mathrm{m}^{2}$, improving the results available in the literature by a factor of more than 4 .
\end{abstract}

Index Terms-terahertz, metasurfaces, biosensor.

\section{INTRODUCTION}

Thanks to the technological advances of the last decades, the Terahertz $(\mathrm{THz})$ band $(0.1-10 \mathrm{THz})$ known as the "THz gap" has become an excellent candidate in the designs of biological sensing devices due to its interesting advantages. The THz radiation is non-ionizing, so the exposure of living organisms to it is non-harmful, allowing to analyze microorganisms in a non-destructive way. In addition, $\mathrm{THz}$ radiation is very sensitive to water, so it is an excellent alternative to perform cancer diagnostic treatments, since cancer cells contain different levels of hydration than healthy cells [1]. Due to these reasons, the $\mathrm{THz}$ band has been proposed for the development of a great variety of biosensing applications, such as detection of bacteria [2] and viruses [3], concentrations of proteins [4], or DNA sequencing [5], among others.

Metamaterials and its planar version, metasurfaces, have also emerged as a new paradigm in biosensing applications. Metasurfaces are planar structures usually made of a composite matrix of subwavelength resonators, able to confine a great electric field concentration in the surroundings of its surface. Thus, they are very sensitive to changes in the refractive index of these areas, making possible the detection of very small amount of samples deposited on the surface. There are many designs based on metasurfaces operating in the $\mathrm{THz}$ regime, with devices capable of detecting very low amounts of yeast [6], viruses [3], [7], or bacteria [8].

Amongst the designs of biosensors based on metasurfaces in the literature, most of them are based on the typical approach of metaatoms, that is, structures that present discrete points in which the electric field is maximum. The main problem with these devices is that the place where the sample is deposited is crucial for its detection, since it is only possible to detect changes in those areas where the electric field is maximum. In order to improve this approach, a design based in a more elaborated geometry was presented recently [9]. Metageometries are designed to have the electric field distributed all along the surface, and not only at discrete points, making these type of structures into a great alternative in the design of biosensing devices, since the place where the samples are deposited becomes no longer so much important.

In this work, a labyrinth metasensor with high sensitivity and Figure of Merit (FOM) is presented. First, a study of the designed structure working as a refractometer is carried out in two different configurations, transmission and reflection, in order to test which of the two options is better for biosensing applications. Then, the final structure is designed and tested as a fungi detector, showing promising results in comparison with other works in the literature.

\section{LABYRINTH METASURFACE: REFRACTOMETER}

(C) 2020 IEEE. Personal use of this material is permitted. Permission from IEEE must be obtained for all other uses, in any current or future media, including reprinting/republishing this material for advertising or promotional purposes, creating new collective works, for resale or redistribution to servers or lists, or reuse of any copyrighted component of this work in other work. 


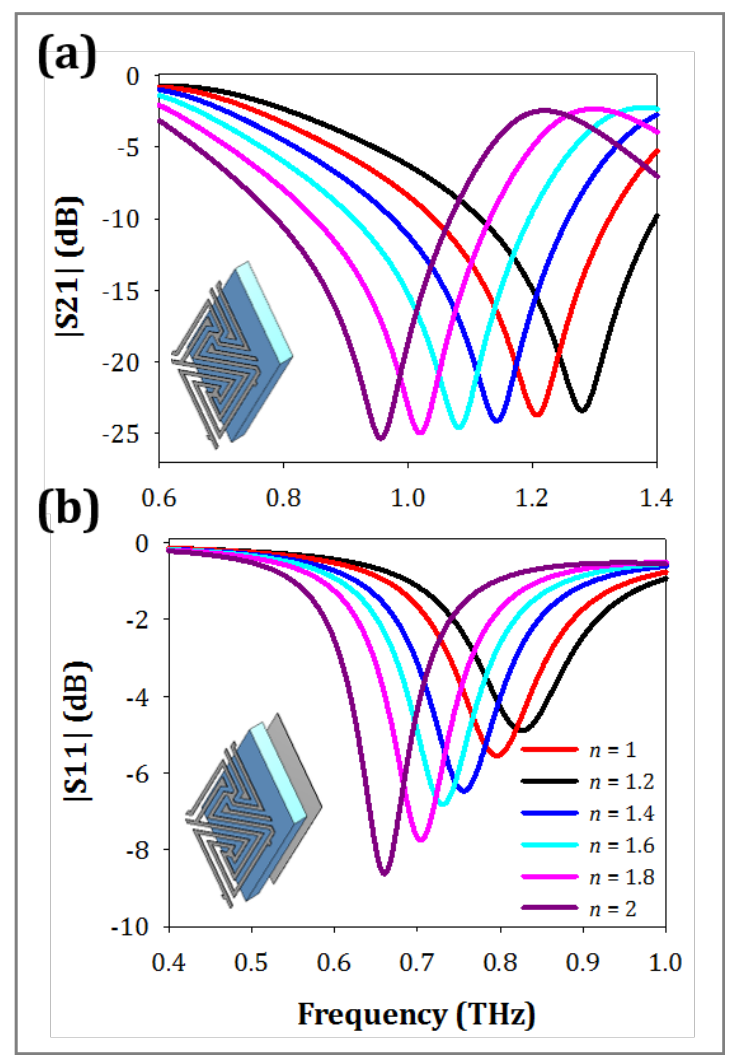

Fig. 1. (a) Transmission coefficient for a thin film of $1 \mu \mathrm{m}$ thicknerss and different refractive indexes, from $n=1$ to $n=2$. Inset: schematic of the unit cell designed. (b) Reflection coefficient for a thin film of $3 \mu \mathrm{m}$ thicknerss and different refractive indexes, from $n=1$ to $n=2$. Inset: schematic of the unit cell designed.

The labyrinth metasurface designed in this work is formed by an aluminum (Al) metallic labyrinth pattern coated over a flexible polypropylene (PP) substrate with the following dimensions: periodicity, $d=36.4 \mu \mathrm{m}$; distance between metallic strips, $s=1.5 \mu \mathrm{m}$; strip width $w=1.5 \mu \mathrm{m}$; metallization thickness $t=0.4 \mu \mathrm{m}$; substrate thickness, $h_{P P}=29 \mu \mathrm{m}$. The structure working in reflection has also a back $\mathrm{Al}$ metallization acting as a ground plane, as shown in the insets of Fig. 1. In the simulation results, obtained with the commercial simulator CST Microwave Studio ${ }^{\circledR}$, shown in the figure, it can be observed that the structure without ground plane presents a stop-band resonance at the frequency of $f_{0, T X}=1.28 \mathrm{THz}$ in transmission, whereas the structure with ground plane presents a reflection dip at the frequency of $f_{0, R X}=0.856 \mathrm{THz}$, for the same dimensions.

In order to study the behavior of both structures as a refractometer, we have deposited an analyte of $3 \mu \mathrm{m}$ thickness and varied its refractive index from $n=1$ to $n=2$. As shown in Fig. 1, the resonance frequency experiences a redshift that increases with the refractive index of the thin film. Concretely, we have achieved a minimum and maximum frequency shift of $74 \mathrm{GHz}(5.8 \%)$ and $325 \mathrm{GHz}$ $(25.4 \%)$ in the transmission configuration, and a minimum and maximum of $44 \mathrm{GHz}(5.14 \%)$ and $208 \mathrm{GHz}(24.3 \%)$ respectively in the reflection configuration. In order to quantify the quality of the device, we have defined the sensitivity as follows: $\mathrm{S}=\Delta f / n$, where $\Delta f=f_{n} f_{0}$, with $f_{n}$ the resonance frequency for each refractive index, $n$, and $f_{0}$ the resonance frequency without the analyte. In addition, the Figure of Merit (FOM) defined as $\mathrm{FOM}=\mathrm{S} / \mathrm{FHWM}$, is a more accurate parameter that also takes into account the spectral linewidth of the curves, making those designs with narrower linewidth better for biosensing purposes, due to the easier detection of discrepancies between different curves. As shown in the previous results, the frequency shift is greater for the transmission configuration. This leads into a maximum sensitivity of $364 \mathrm{GHz} / \mathrm{RIU}$ (where RIU stands for refractive index units), in comparison with the $220 \mathrm{GHz} / \mathrm{RIU}$ obtained in the reflection configuration. Nevertheless, if we observe the curves at Fig. 1, we can notice that although this shift is larger for the transmission structure (panel (a)), the bandwidth of the curves in the reflection structure (panel (b)) are narrower, giving rise to a higher FOM(average value of $1.65 \mathrm{RIU}^{-1}$ versus 0.57 $\mathrm{RIU}^{-1}$ ), and making the structure working in reflection configuration a better option to design biosensing devices.

\section{LABYRINTH METASURFACE: FUNGI DETECTOR}

In this section the behavior of the structure working is transmission as a fungi detector has been numerically studied in the band of $0.6-1 \mathrm{THz}$. Fungi have been represented as dielectric cylinders with a radius $r_{f}=2 \mu \mathrm{m}$, height $h_{f}=1 \mu \mathrm{m}$ and dielectric permittivity of $\varepsilon_{f}=8$ [2] and have been arbitrarily deposited on the surface of the structure with a randomizing function.

First, a statistical study is carried out in order to check the repeatability of the different measures. Ten different simulations were carried out for each fungi concentration, between $N=5$ and $N=100$, where $N$ is the number of fungi elements, and represents a fractional area between $5.2 \%$ and $51.1 \%$ respectively. The fractional area has been calculated as $A_{f}=A_{o} / A_{T}$, where $A_{o}$ represents the area occupied by the microorganisms (extracted directly from CST), and $A_{T}$ the total area of the metasensor surface. Depending on the fungi overlap $A_{o}$ presents slight variations. Therefore, we have taken an average of all the cases. Fig. 2 (a) shows a front view of the structure with the fungi elements spread over the surface (top), and the resonance frequency for each concentration (bottom).

Although there exist slight variations in each simulation that are caused by the fungi positions in the unit cell, detection thresholds that avoid overlap between consecutive concentrations can be defined. To get a better view of the structure performance, we have taken the simulations whose resonance frequency was closest to the mean resonance frequency for each $N$ and represented the frequency shift in Fig. 2(b). The minimum frequency shift is $1.6 \%$ for the case of $N=5$, with a standard deviation of $8.5 \mathrm{GHz}$. The maximum shift is for the case of $N=100$ and reaches $19 \%$ with a standard deviation of $10.3 \mathrm{GHz}$.

These values reveal that the structure designed is able to detect very low concentrations of microorganisms deposited 


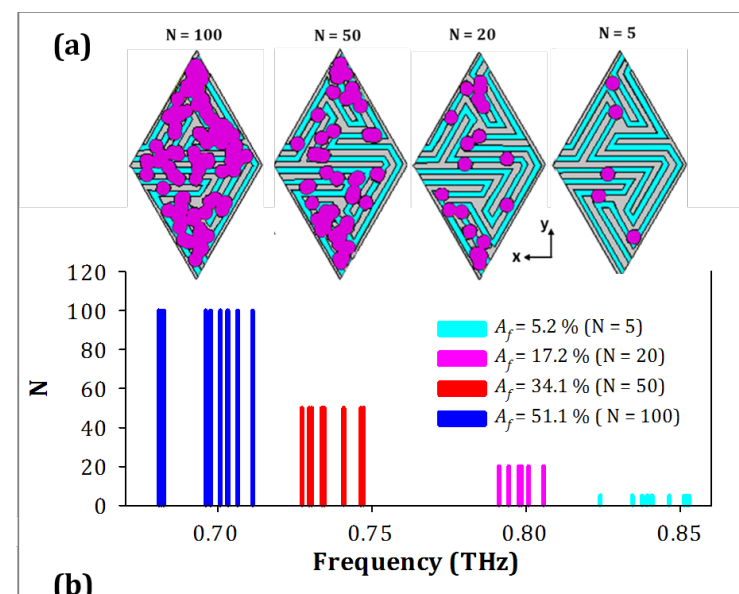

(b)

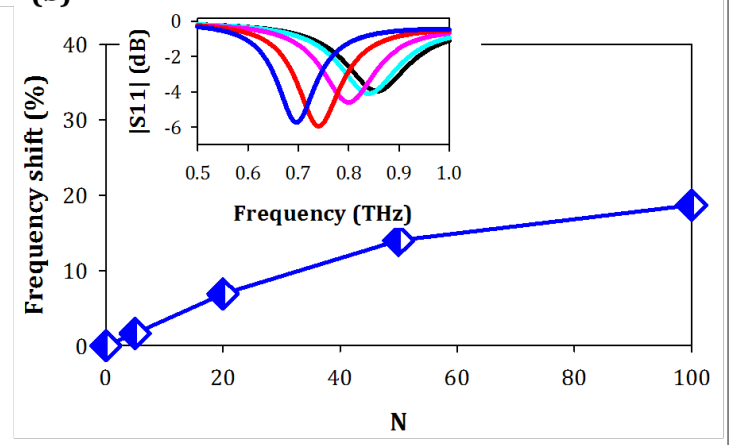

Fig. 2. (a) Front view of the designed structure with $N=5 ; 20 ; 50 ; 100$ fungi(purple cylinders) (top) and resonance frequency for all the simulations carried out for different fungi concentrations (bottom). (b) Frequency shift as a function of the fungi concentration. Inset: reflection coefficient for different fungi concentrations, cases nearest to the mean.

on its surface. With these results, we can calculate again de sensitivity and FOM of the structure. In this case, the

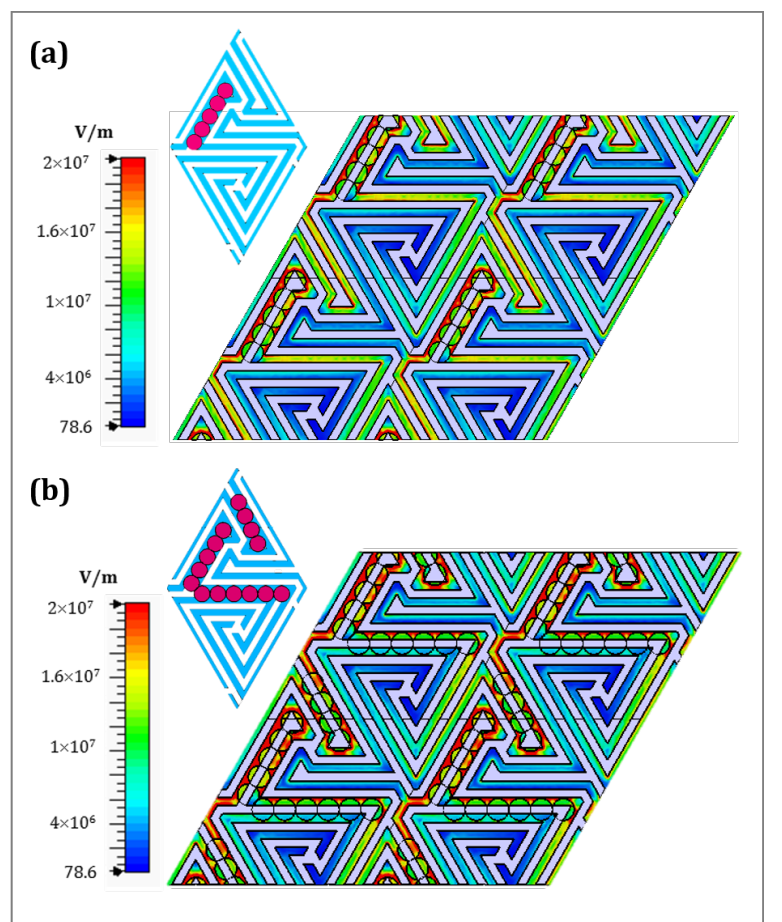

Fig. 3. (a) Electric field distribution over the labyrinth surface for the designed structure for a fractional area of $5.48 \%(N=5)$ and schematic of the fungi elements distribution on the unit cell. (b) The same as (a) for a fractional area of $16.45 \%(N=15)$ sensitivity has been calculated as a function of the fractional area and the refractive index, as follows: $\mathrm{S}=\Delta f / A \cdot n$ where $\Delta f=f_{N} f_{0}$, with $f_{N}$ the resonance frequency for each fungi concentration, $N$, and $f_{0}$ the resonance frequency without the analyte; $A_{f}$ the fractional area occupied by the fungi; and $n$ the refractive index of the fungi. The mean sensitivity achieved with these definitions is $80 \mathrm{GHz} / \mathrm{RIU}$ and the mean FOM is $0.85(\mathrm{RIU})^{-1}$. The specific results for each concentration are shown at Table 1 . In order to make a comparison with other similar work found in the literature, we have chosen the study presented in [2], where a metasurface consisting in a square ring with a capacitive gap at the center was used for fungi detection. In that work, a frequency shift of $1 \%$ for an arbitrary fungi deposition at a minimal density of $0.09 / \mu \mathrm{m}^{2}$ was observed, where the density was calculated as ratio between the average fungi elements in the metasurface capacitive gap and the gap area. In our case, we have deposited microorganisms at a density of $0.004 / \mu \mathrm{m}^{2}$ in the case of having $N=5$ fungi elements and calculating the ratio between the total number of elements and the total area of the surface, obtaining a frequency shift of $1.6 \%$, and thus achieving a larger frequency shift with a lower density than in the previous work.

\section{MAXIMIZING THE PERFORMANCE}

In order to find the maximum performance of the device and motivated by the work presented in [2], we deposited $N=5$ microorganisms in the area of the device that presented the highest electric field concentration, as shown in Fig. 3(a). With such a small amount of sample, we obtained a frequency shift of $77 \mathrm{GHz}$ that corresponds with $9 \%$ variation, more than the value achieved in the case of depositing $N=20$ fungi elements in an arbitrary manner. This is explained due to the intricated geometry of this structure, that contrary to other classical designs based on a metaatom approach with discrete hotspots of electric field confinement, has the electric field confinement distributed all along the surface of the structure. As can be shown at Fig. 3(c) there are more areas with a high electric field confinement. We have coated these areas with $N=15$ fungi elements and the frequency shift that we have obtained for a fractional area of $16.45 \%$ is $134 \mathrm{GHz}(15.6 \%)$, a variation almost similar to the case of having $N=100$ elements (see Table 1), demonstrating the importance of the sample localization, and the advantage in the use of designs based on metageometries, where the electric field is more distributed than in the classical metaatom approach, and thus the place where the sample falls is not so critical. 
a. Cases corresponding to an ordered distribution of the fungi elements in the surface areas where the electric field is maximum

\section{ACKNOWLEDGMENT}

Work funded by project RTI2018-094475-B-I00 (MCIU/AEI/FEDER, UE)

\section{REFERENCES}

[1] E. Pickwell and V. P. Wallace, "Biomedical applications of terahertz technology," J. Phys. D. Appl. Phys., vol. 39, no. 17, pp. R301-R310, 2006.

[2] S. J. Park et al., "Detection of microorganisms using terahertz metamaterials.," Sci. Rep., vol. 4, p. 4988, 2014.

[3] S. J. Park, S. H. Cha, G. A. Shin, and Y. H. Ahn, "Sensing viruses using terahertz nano-gap metamaterials," Biomed. Opt. Express, vol. 8, no. 8, p. 3551, 2017.

[4] L. Xie, W. Gao, J. Shu, Y. Ying, and J. Kono, "Extraordinary sensitivity enhancement by metasurfaces in terahertz detection of antibiotics," Sci. Rep., vol. 5, p. 8671, 2015.

[5] Y. Yang, D. Xu, and W. Zhang, "High-sensitivity and label-free identification of a transgenic genome using a terahertz metabiosensor," Opt. Express, vol. 26, no. 24, p. 31589, 2018.

[6] S. J. Park, B. H. Son, S. J. Choi, H. S. Kim, and Y. H. Ahn, "Sensitive detection of yeast using terahertz slot antennas," Opt. Express, vol. 22, no. 25, p. 30467, 2014.

[7] J. T. Hong et al., "Enhanced sensitivity in THz plasmonic sensors with silver nanowires," Sci. Rep., vol. 8, no. 1, pp. 1-8, 2018.

[8] S. J. Park et al., "Detection of microorganisms using terahertz metamaterials.," Sci. Rep., vol. 4, p. 4988, Jan. 2014.

[9] I. Jáuregui-López, P. Rodriguez-ulibarri, A. Urrutia, S. A. Kuznetsov, and M. Beruete, "Labyrinth Metasurface Absorber for Ultra-High-Sensitivity Terahertz Thin Film Sensing," Phys. status solidi - Rapid Res. Lett., vol. 12, no. 10, p. 1800375, Oct. 2018.

\begin{tabular}{|c|c|c|c|c|c|c|c|}
\hline $\mathbf{N}$ & 0 & 5 & 20 & 50 & 100 & $5^{a}$ & $15^{\mathrm{a}}$ \\
\hline$A_{f}(\%)$ & - & 5.23 & 17.2 & 34.1 & 51.1 & 5.48 & 16.4 \\
\hline$f_{r e s}(\%)$ & - & 1.64 & 6.85 & 13.9 & 18.7 & 9.02 & 15.6 \\
\hline $\begin{array}{l}\text { Standard } \\
\text { Deviation }\end{array}$ & - & 8.5 & 5 & 7.5 & 10.3 & - & - \\
\hline $\begin{array}{l}\text { Sensitivity } \\
\text { (GHz/RIU) }\end{array}$ & - & 67.3 & 85.2 & 87.6 & 78.2 & 352 & 203 \\
\hline FOM $(\text { RIU })^{-1}$ & - & 0.73 & 0.89 & 0.92 & 0.87 & 2 & 1.36 \\
\hline
\end{tabular}

